Conclusion The prevalence of VRE catheter- associated urinary tract infections among NICU patients and Pediatric ward has been rare in Tehran. PFGE results revealed that among enterococci isolates PFGE patterns were diverse.

\section{PREVALENCE OF PANDEMIC (H1N1) AND SEASONAL INFLUENZA VIRUS INFECTION IN PEDIATRIC AGE GROUP IN SOUTHERN IRAN}

doi:10.1136/archdischild-2012-302724.0877

A Moattari, A Emami. Bacteriology \& Virology, Shiraz University of Medical Sciences, Shiraz, Iran

Background A novel strain of H1N1 Influenza (A/California/7/2009) has been prevalent since April 2009. Epidemiological and clinical studies showed that children at high risk for severe infection. Influenza virus infections are associated with significant morbidity and mortality in children with acute respiratory infections.

Aim To determined the prevalence of new H1N1 and seasonal influenza viruses in children with fever, cough, bronchiolitis, pneumonia wheezing and asthma symptoms.

Methods Pharyngeal swabs were taken from 450 children aged (1-60 months) with respiratory tract symptom between June 2009 to March 2012. The specimens were tested using Real Time Reverse Transcriptase PCR.

Results Out of 450 samples 130 were tested positive for pandemic $\mathrm{H} 1 \mathrm{~N} 1,75 \mathrm{H} 3 \mathrm{~N} 2$ and 15 influenza type B infections.

Conclusion According to our results $28.8 \%$ of respiratory infection in children in the south of Iran was due to new H1N1, 16.7\% H3N2, $3.3 \%$ B Influenza viruses during the foregoing pandemic. Our analysis revealed no significant correlation between males and females.

\section{THE PREVALENCE OF HUMAN BOCAVIRUS AND ADENOVIRUS IN HOSPITALIZED CHILDEREN IN SHIRAZ- IRAN}

doi:10.1136/archdischild-2012-302724.0878

A Emami, A Moattari, N Pirbonyeh. Bacteriology \& Virology, Shiraz University of Medical Sciences, Shiraz, Iran

Background Acute respiratory infection is common cause of morbidity and mortality especially during childhood. The most important viral respiratory infection, such as: Influenza viruses, Adeno virus, Respiratory syncytial virus and Bocaviruses are the most common infections in upper and lower respiratory tract diseases. Different studies show Adenoviruses and $H B O V$ are the most important potential respiratory pathogen after influenza virus with the same manifestations. $H B o V$ in the parvovirus family first was detected in 2005 by molecular methods.

Aim In this study we tried to evaluate the prevalence of $H A d V$ and $H B \circ V$ in patients with respiratory tract infection.

Materials and Methods In this cross sectional study for two years (2009-2010), 150 nasopharyngeal swabs collected from youth age $(\leq 15)$ with acute upper respiratory tract syndromes referring to the Shiraz University of Medical Hospitals. Adenoviruses were detected by nested-PCR and bocavirus was detected by usual conventional PCR with specific primers.

Results Among the 150 taken samples, 40 samples (27\%) was positive for adenovirus and in 1 sample $(0.66 \%) \mathrm{HBoV}$ was amplified which was co-infected with adenovirus.

Conclusion The result of this study showed that Adenoviruses have more prevalence in south of Iran compare to influenza viruses during this two years, but Bocavirus has no more prevalence in this region between this groups of patients. Up to the result of this study it has been recommended for optimizing the treatment of this group of patients, other viral respiratory infections must be evaluated.

\section{CHARACTERISTICS OF CHILDREN'S LEICHMANIASIS IN MONTENEGRO}

doi:10.1136/archdischild-2012-302724.0879

B Andric, D Terzic, B Dupanovic, D Nikcevic. Clinic for Infectious Diseases, Clinic Center of Montenegro, Podgorica, Montenegro

Background Montenegro is an endemic area for visceral leishmaniasis. Natural condition and geographical position (Mediterranean area) allow of visceral leishmaniasis existence. Epidemiological studies warn on the increase in number of disease in Montenegro, which are 3 patients on 646000 inhabitants per year.

Methods The investigations used epidemiological, clinical and laboratory methods.

Diagnosis was etiologically confirmed through bone marrow bioptate analysis, by direct microscoping of serial sections colored with the Giemsa s stain, Reticulin (Gordon and Sweet method), PAS method, and by immune-biochemical methods, and serological method.

Results In the period from 1992 to 2011 in Montenegro, 67 cases of leishmaniasis have been diagnosed, with 4 lethal outcomes. The children population participate with $36(\%)$ cases, age $0-4$ years in $22(40 \%)$ and age $5-15$ years in $14(\%)$. Which the prevalence of general infective syndrome (high febricity, exhaustion), in all children cases, enlarged of spleen in 28 cases, and liver in 26 cases, anemia in 29 cases, pancytopenia in 22 cases, increased serum transaminases in 27 cases. Recidives were registered in 9 patients. According to the geographic area where the disease was diagnosed the expansion include the entire coastal area of Montenegro from Ulcinj to Herceg Novi, Cetinje, the area of Skadar lake including Podgorica and even some northern parts of Montenegro.

Conclusion Expansion of the primary endemic focus, more severe types of the disease, co-infective types and increase in mortality, therapy resistance, coinfective forms of disease are the new features of leishmaniasis in Montenegro.

\begin{tabular}{l}
\hline 880 THE RESISTANCE OF S. AUREUS TO ANTIBACTERIALS IN \\
CHILDREN
\end{tabular}

doi:10.1136/archdischild-2012-302724.0880

'OA Nazarchuk, ${ }^{2} \mathrm{GG}$ Nazarchuk, ${ }^{3} \mathrm{DV}$ Paliy, ${ }^{4} \mathrm{DV}$ Dmytriiev. 'Microbiology, Virology and Immunology; ${ }^{2}$ Ophtalmology and Eye Diseases; ${ }^{3}$ Infectious Diseases; ${ }^{4}$ Anesthesiology and Intensive Care, Vinnitsa N. Pyrogov Memorial National Medical University, Vinnitsa, Ukraine

Background and Aims Clinical strains of S. aureus are one of the most widely spread microorganisms, causing nosocomial purulent infections in pediatric anesthesiology practice. Treatment of infectious diseases caused by Staphylococcus is a difficult task in conditions of developing resistance to antibiotics.

Methods Our research work is dedicated to the problem of antibiotic resistance of $\mathrm{S}$. aureus clinical strains, obtained from children with purulent-inflammatory diseases. The Staphylococcus strains' sensitivity to the spectrum of antibiotics, nowadays widely used in clinical practice, was studied by means of disc-diffusion method.

Results The results of the study show high resistance of S. aureus to antibiotics of penicillin (26.79-53.57\%). About $42.86 \%$ of strains were resistant to oxacillin. It proves the presence of methicillinresistant strains of $\mathrm{S}$. aureus in children with purulent diseases. S. aureus had also low sensitivity to ureidopenicillins, aminopenicillins, carbenicillins. As for aminopennicilin with clavulan acid only $1.78 \%$ of strains were resistant. The 1st (cafazolin-83.93\%) and the 3rd generation of cephalosporins (ceftriaxone- $89.28 \%$, ceftazidim- $80.36 \%$ ) had high activity against S. aureus. Obtained isolates of S. aureus were sensitive to meropenem (87.5\%) aminoglycosides (14.29-89.29\%). 67.86\% of strains were sensitive to rifampicin. But only in $41.07 \%$ of cases S. aureus was sensitive to vancomycin. Doxycycline was effective in $57.14 \%$. 
Conclusions In our study S. aureus strains obtained from children purulent-infectious diseases have high sensitivity to aminopenicillin with clavulan acid, meropenem, some cephalosporins, gentamycin. Among clinical strains of S. aureus we found methicillin- and even vancomycin-resistant microorganisms.

\section{1 \\ A RARE CASE OF LEMIERRE SYNDROME IN A 13 YEAR OLD CHILD}

doi:10.1136/archdischild-2012-302724.0881

II Velissariou, ${ }^{2} \mathrm{P}$ Papapavlou, ${ }^{2} \mathrm{H}$ Bairactaris, ${ }^{3}$ | Pierris, ${ }^{3} \mid$ Kolovos, ${ }^{1} \mathrm{M}$ Kalmanti. 'Pediatrics; ${ }^{2}$ Vascular Surgery; ${ }^{3}$ Radiology, Metropolitan Hospital, Athens, Greece

Object To present a rare case of Lemierre syndrome in a 13 year old girl.

Case Report The child presented with pyrexia, pharyngitis and dysphagia of 9 days duration. On the fifth day of pyrexia she developed a painful mass on the left side of her neck with bilateral tonsillar enlargement with exudate.

The patient underwent cervical ultrasound and magnetic resonance angiography of the brain that revealed septic thrombophlebitis of the left internal jugular vein. Antibody testing for cytomegalovirus, Toxoplasma gondii and Bartonella henselae were negative for active infection. Blood cultures did not have any growth. Computed tomography of the chest revealed multiple bilateral septic emboli, although the patient did not have any overt respiratory symptoms.

The patient received intravenous ceftriaxone and clindamycin for 3 weeks, followed by amoxicillin-clavoulanic acid orally for another 3 weeks along with anticoagulation therapy for 3 months in total.

Three months later, she was clinically asymptomatic, computed tomography of the chest was clear and the thrombophlebitis of the left internal jugular vein was stable.

Almost two years later, the patient remains in a very good clinical condition without any similar recurrences.

Conclusions Lemierre syndrome is a rare combination of tonsillitis and septic thrombophlebitis of the internal jugular vein caused primarily by Fusobacterium necrophorum, an obligate anaerobic gram-negative rod. In our case, we did not isolate the causative agent, however the patient had an excellent outcome with antibiotic and anticoagulation therapy without any surgical intervention.

\section{HEPATOSPLENIC CAT-SCRATCH DISEASE IN A 12-YEARS- OLD GIRL}

doi:10.1136/archdischild-2012-302724.0882

0 Diaz-Morales, JD Martinez-Pajares, JC Ramos-Diaz, J Trigo-Moreno, E FernandezGomez, M Miranda-Valdivieso. UGC Pediatria, Hospital Comarcal de Antequera, Antequera, Spain

Background Cat-scratch disease (CSD) is an infectious disease typically characterized by a self-limited regional lymphadenopathy. However, CSD can include hepatic and splenic involvement. There are few data in the literature regarding treatment of this situation, although administration of rifampicin associated with gentamicin and doxycycline or azithromycin is promoted.

Case Report A 12 year old girl was admitted because of 3 weeks sustained fever. No remarkable physical findings were presents. Laboratory findings in this admission only show CRP and ESR elevation and positive IgM for cytomegalovirus (CMV), together with fever defervescence, so she was discharged with probable diagnosis of acute CMV infection. However, she was readmitted a week later for recurrence of fever. Abdominal ultrasound detected multiples hyperechoic hepatic lesions and two larger splenic lesions, with no abnormalities of liver function. IgM (1/192) and IgG (1/3200) were positive for Bartonella henselae and PCR to CMV was negative, getting the diagnosis of an hepatosplenic form of CSD. Treatment with rifampicin and trimetroprim during 14 days was unsuccessful, therefore, triple therapy with rifampicin, doxycycline and azithromycin was started. Fever stopped after 6 days of treatment, but reemerged a week later, with a rebound of CRP and ESR levels. Finally, fever and analytical anomalies disappeared after several weeks, under monotherapy with azithromycin. No immunodefienciency was found.

Comments CSD must be suspected in the presence of prolonged fever with or without hepatosplenic involvement. In this case, little response was observed to the antibiotic therapy suggested in the literature, and evolution appeared to be self-limited.

\section{INCIDENCE OF LEISHMANIASIS IN A DISTRICT GENERAL HOSPITAL}

doi:10.1136/archdischild-2012-302724.0883

1,2S Damodaran, ${ }^{3} \mathrm{G}$ Erumbala, ${ }^{4} \mathrm{D}$ Abraham, 'S Nirmal. 'Paediatrics, James Paget Hospital, Great Yarmouth; 'PICU, Addenbrookes Hospital, Cambridge; ${ }^{3}$ Paediatrics, Milton Keynes General Hospital NHS Foundation Trust, Milton Keynes; ${ }^{4} \mathrm{NICU}$, Birmingham Children's Hospital, Birmingham, UK

Aim To study the incidence of Leishmaniasis in children under 5 years in a District General Hospital in the UK.

Method The microbiology records over 5-years (2005-2010) were reviewed. Inclusion criteria were age under 5 years and diagnosis of Leishmaniasis on bone marrow examination.

Results Two cases were identified.

Case-1: A 15 month-old-girl, family from east Timur, referred from primary care with weight-loss and a non-healing skin ulcer. She appeared undernourished with pallor, pyrexia and hepatosplenomegaly. FBC showed pancytopenia. Bone marrow examination confirmed Leishmaniasis. Her mother had intrapartum Leishmaniasis.

Case-2: A 22 month-old-boy presented with high fever and weight loss for 3 weeks. Examination confirmed pyrexia, pallor and hepatosplenomegaly. FBC revealed pancytopenia. Direct antigen test for Leishmania was positive. Leishmania Donovani complex was detected on bone marrow examination. There was no history of maternal infection.

Both children were born in United Kingdom with no history of foreign travel. They both responded well to treatment with ambisome.

Discussion Leishmania Donovani, the protozoan parasite, is transmitted in endemic areas by the insect vector Phlebotomine sandfly. In non-endemic areas, transmission is vertical. The infection can lead to impairment in cell mediated immunity and a 100\% mortality rate, if left untreated. Most cases of visceral Leishmaniasis occur in India, Bangladesh, Nepal, Brazil, and the Sudan. East Timor, although not endemic, has witnessed an upsurge of several confirmed cases recently. There was a history of maternal infection in only one of these two children. The source of infection in the other remains unidentified.

\section{THE VARIABILITY IN THE COURSE OF HEPATITIS B IN CHILDREN AFTER MOTHER-TO-CHILD TRANSMISSION AS A REASON TO ADJUST TREATMENT}

doi:10.1136/archdischild-2012-302724.0884

1.2B Kowalik-Mikołajewska, 1,2M Aniszewska, 1,2M Marczyńska. 'Department of Children's Infectious Diseases, Medical University of Warsaw; ${ }^{2}$ Hospital of Infectious Diseases, Warsaw, Poland

Background and Aims In Poland currently, despite obligatory hepatitis $B$ vaccination we expect the occurence of mother-to-child 